\title{
Severe cognitive impairment in a patient with CMT2A
}

Pedro J. Tomaselli, ${ }^{1}$, Mahima Kapoor ${ }^{1}$, Andrea Cortese ${ }^{1}$, James M. Polke ${ }^{3}$, Alexander M. Rossor ${ }^{1}$, and Mary M. Reilly

1. MRC Centre for Neuromuscular Diseases and National Hospital for Neurology and Neurosurgery, UCL Institute of Neurology, London, UK

2. Department of Neuromuscular Disorders, Clinical Hospital of Ribeirão Preto, University of São Paulo, Ribeirão Preto, SP, Brazil

3. Department of Neurogenetics, The National Hospital for Neurology and Neurosurgery, UCL Institute of Neurology, London, UK

Dear Editor,

Mutations in mitofusin 2 (MFN2) are the most common cause of axonal Charcot Marie Tooth disease (CMT2) (Polke; 2011). Additional features known to occasionally occur with MFN2-related disorders are optic atrophy, pyramidal signs, scoliosis and deafness (Zuchner, 2006; Feely, 2011; Bombelli, 2014). Cognitive impairment has been reported in a small number of patients with MFN2 mutations (Genari, 2011; Tufano, 2015).(PMID 18946002)

We report a patient with a c.310C>T; p.R104W mutation in MFN2 with severe cognitive involvement. The patient (aged 22) is the youngest daughter of five siblings from healthy and unrelated parents of Eastern European origin and is the only affected person in her family. She had delayed motor development, walking by three years of age. Her neuropathy presented in a length-dependent fashion but with significant 
proximal involvement. She required splints from 4 to 8 years of age, a walker between age 8 and 13 years, and has used a wheelchair since 13 years of age. In addition to her neuropathy, her language was significantly delayed. She first spoke words at age five to six and at age 22, speech was limited to short sentences. She could read simple sentences, recite the alphabet, and name basic animals and common objects. Orientation was limited to name, age, and place but not time. She could accurately count objects dispersed in space and name colors. She had formal cognitive testing at age 14 which reported abilities equivalent to a 3-4 year old in some cognitive domains.

Examination at 22 years of age revealed both proximal and distal wasting and weakness in the upper (UL) and lower limbs (LL) with MRC scores of 5 proximally and 0 distally in the UL, and 4- proximally and 0 distally in the LL. She was areflexiclt was not possible to do a sensory examination. There was evidence of bilateral optic atrophy, visual acuities were $6 / 24$ in the right eye and 6/18 on the left.

A nerve conduction study performed at eight years of age revealed an axonal neuropathy. There was a marked reduction of the compound muscle action potentials (CMAP) of the median $(0.6 \mathrm{mV})$ and ulnar $(0.4 \mathrm{mV})$ nerves, which had a conduction velocity of $35.1 \mathrm{~m} / \mathrm{s}$. A MRI scan of the brain at 4 years of age revealed widened prepontine cisterns (Figure 1). The p.R104W mutation has previously been reported in association with learning difficulties (Del Bo et al., 2008; Genari et al., 2011; Tufano et al., 2015).

We describe a patient with severe global developmental delay, providing additional evidence that the p.R104W mutation in MFN2 (unlike other MFN2 mutations) may 
cause cognitive impairment in addition to an axonal neuropathy. The molecular mechanisms by which the p.R104W mutation results in cognitive impairment is unknown.

\section{References}

Bombelli F, Stojkovic T, Dubourg O, et al. Charcot-Marie-Tooth disease type 2A: from typical to rare phenotypic and genotypic features. JAMA Neurol 2014;71:1036-1042.

Del Bo R, Moggio M, Rango M, et al. Mutated mitofusin 2 presents with intrafamilial variability and brain mitochondrial dysfunction. Neurology 2008;71:1959-1966.

Feely SM, Laura M, Siskind CE, et al. MFN2 mutations cause severe phenotypes in most patients with CMT2A. Neurology 2011;76:1690-1696.

Genari AB, Borghetti VH, Gouvea SP, et al. Characterizing the phenotypic manifestations of MFN2 R104W mutation in Charcot-Marie-Tooth type 2. Neuromuscul Disord 2011;21:428-432.

Polke JM, Laura M, Pareyson D, et al. Recessive axonal Charcot-Marie-Tooth disease due to compound heterozygous mitofusin 2 mutations. Neurology 2011;77:168-173.

Tufano M, Cappuccio G, Terrone G, et al. Early onset Charcot-Marie-Tooth neuropathy type 2A and severe developmental delay: expanding the clinical phenotype of MFN2-related neuropathy. J Peripher Nerv Syst 2015;20:415-418.

Zuchner S, De Jonghe P, Jordanova A, et al. Axonal neuropathy with optic atrophy is caused by mutations in mitofusin 2. Ann Neurol 2006;59:276-281.

Figure 1. A sagittal T1 weighted MRI demonstrating wide prepontine cisterns 